\title{
Pentaquarks in the Jaffe-Wilczek approximation
}

\author{
I.M.Narodetskii ${ }^{1)}$, C.Semay ${ }^{2)}$, \\ B.Silvestre-Brac ${ }^{3)}$, Yu.A.Simonov ${ }^{1)}$, M.A.Trusov ${ }^{1)}$
}

\begin{abstract}
The masses of $u u d d \bar{s}, u u d d \bar{d}$ and $u u s s \bar{d}$ pentaquarks are evaluated in a framework of both the Effective Hamiltonian approach to QCD and spinless Salpeter using the Jaffe-Wilczek diquark approximation and the string interaction for the diquarkdiquark-antiquark system. The pentaquark masses are found to be in the region above $2 \mathrm{GeV}$. That indicates that the Goldstone boson exchange effects may play an important role in the light pentaquarks. The same calculations yield the mass of $[u d]^{2} \bar{c}$ pentaquark $\sim 3250 \mathrm{MeV}$ and $[u d]^{2} \bar{b}$ pentaquark $\sim 6509 \mathrm{MeV}$.
\end{abstract}

1) Institute of Theoretical and Experimental Physics, Moscow, Russia.

2) Groupe de Physique Nucléaire Théorique, Université de Mons-Hainaut, Académie universitaire Wallonie-Bruxelles, Mons, Belgium.

3) Laboratoire de Physique Subatomique et de Cosmologie, Grenoble-Cedex, France. 


\section{Introduction}

Recently LEPS and DIANA collaborations [1, 2] reported the observation of a very narrow peak in the $K^{+} n$ and $K^{0} p$ invariant mass distribution which existence has been confirmed by several experimental groups in various reaction channels [3]. These experimental results were motivated by the pioneering paper on chiral soliton model [4]. The reported mass determinations for the $\Theta$ are very consistent, falling in the range $1540 \pm 10$, with the width smaller than the experimental resolution of $20 \mathrm{MeV}$ for the photon and neutrino induced reactions and of $9 \mathrm{MeV}$ for the ITEP $K^{+} \mathrm{Xe} \rightarrow K^{0} p \mathrm{Xe}^{\prime}$ experiment.

From the soliton point of view the $\Theta$ is nothing exotic compared with other baryons - it is just a member of $\overline{\mathbf{1 0}}_{F}$ multiplet with $S=+1$. However, in the sense of the quark model $\Theta^{+}(1540)$ baryon with positive amount of strangeness is manifestly exotic - its minimal configuration can not be satisfied by three quarks. The positive strangeness requires an $\bar{s}$ and $q q q q$ (where $q$ refers to the lightest quarks $(u, d)$ ) are required for the net baryon number, thus making a pentaquark uudd $\bar{s}$ state as the minimal "valence" configuration. Later NA49 collaboration at CERN SPS [5] announced evidence for an additional narrow udus $\bar{s}$ resonance with $I=3 / 2$, a mass $1.862 \pm 0.002 \mathrm{GeV}$ and a width below the detector resolution of about $18 \mathrm{MeV}^{1)}$ and $\mathrm{H} 1$ collaboration at HERA [6] found a narrow resonance in $D^{*-} p$ and $D^{*+} \bar{p}$ invariant mass combinations at $3.099 \pm 0.033_{\text {stat }} \pm 0.005_{\text {syst }} \mathrm{GeV}$ and a measured Gaussian width of $12 \pm 3_{\text {stat }} \mathrm{MeV}$, compatible with the experimental resolution. The later resonance is interpreted as an anti-charmed baryon with a minimal constituent quark composition of uudd $\bar{c}$, together with the charge conjugate. The discoveries of first manifestly exotic hadrons mark the beginning of a new and rich spectroscopy in QCD and provide an opportunity to refine

\footnotetext{
${ }^{1)}$ NA49 also reports evidence for a $\Xi^{0}(1860)$ decaying into $\Xi(1320) \pi$.
} 
our quantitative understanding of nonperturbative QCD at low energy.

The $\Theta$-hyperon has hypercharge $Y=2$ and third component of isospin $I_{3}=0$. The apparent absence of the $I_{3}=+1, \Theta^{++}$in $K^{+} p$ argues against $I=1$, therefore it is usually assumed the $\Theta$ to be an isosinglet. The other quantum numbers are not established yet.

As to the theoretical predictions we are faced with a somewhat ambiguous situation, in which exotic baryons may have been discovered, but there are important controversies with theoretical predictions for masses of pentaquark states. The experimental results triggered a vigorous theoretical activity and put a renewed urge in the need to understand of how baryon properties are obtained from QCD.

All attempts of the theoretical estimations of the pentaquark masses can be subdivided into following four categories: (i) dynamical calculations using the sum rules or lattice QCD [7, 8], (ii) the phenomenological analyses of the hyperfine splitting in quark model [9, 10], (iii) phenomenological analyses of the $S U(3)_{F}$ mass relations, and (iv) dynamical calculations using the chiral $S U(3)$ quark model [11]).

The QCD sum rules predict a negative parity $\Theta^{+}$of mass $\simeq 1.5 \mathrm{GeV}$, while no positive parity state was found [7]. The lattice QCD study also predicts that the parity of the lowest $\Theta$ hyperon is most likely negative [8].

The naive quark models, in which all constituents are in a relative $S$ wave, naturally predict the ground state energy of a $J^{P}=\frac{1}{2}^{-}$pentaquark to be lower than that of a $J^{P}=\frac{1}{2}^{+}$one. However, using the arguments based on both the Goldstone boson exchange between constituent quarks and color-magnetic exchange it was mentioned that the increase of hyperfine energy in going from negative to positive parity states can be quite enough to compensate the orbital excitation energy $\sim 200 \mathrm{MeV}$. 
However, existing dynamical calculations of pentamasses using the chiral $S U(3)$ quark model (see, e.g., [11]) are subject to significant uncertainties and can not be considered as conclusive.

Pentaquark baryons are unexpectedly light. Indeed, a naive quark model with quark mass $\sim 350 \mathrm{MeV}$ predicts $\Theta^{+}$at about $350 \times 5=1750$ $\mathrm{MeV}$ plus $\sim 150 \mathrm{MeV}$ for strangeness plus $\sim 200 \mathrm{MeV}$ for the $P$-wave excitation. A natural remedy would be to decrease the number of constituents. This leads one to consider dynamical clustering into subsystems of diquarks like $[u d]^{2} \bar{s}$ [12] and/or triquarks like $[u d][u d \bar{s}][10]$ which amplify the attractive color-magnetic forces. In particular, in [12] it has been proposed that the systematics of exotic baryons can be explained by diquark correlations.

The quark constituent model have not been yet derived from QCD. Therefore it is tempting to consider the Effective Hamiltonian (EH) approach in QCD (see, e.g., [13]) which from one side can be derived from QCD and from another side leads to the results for the $\bar{q} q$ mesons and $3 q$ baryons which are equivalent to the quark model ones with some important modifications. The EH approach contains the minimal number of input parameters: current (or pole) quark masses, the string tension $\sigma$ and the strong coupling constant $\alpha_{s}$, and does not contain fitting parameters as, e.g., the total subtraction constant in the Hamiltonian. It should be useful and attractive to consider expanding of this approach to include diquark degrees of freedom with appropriate interactions. The preview of this program was done in [14]. It is based on assumption that chiral and the short range gluon exchange forces are responsible for the formation of $u d$ diquarks in $\Theta$ while the strings are mainly responsible for binding constituents in $\Theta$. In this paper we review and extend application of the EH approach to the Jaffe-Wilczek model of pentaquarks.

In this model inside $\Theta(1540)$ and other $q^{4} \bar{q}$ baryons the four quarks are 
bound into two scalar, singlet isospin diquarks. Diquarks must couple to $\mathbf{3}_{c}$ to join in a color singlet hadron. In the quark model five quarks are connected by seven strings. In the diquark approximation the short legs on this figure shrink to points and the five-quark system effectively reduces to the three-body one, studied within the EH approach in [15, 16]. In total there are six flavor symmetric diquark pairs $[u d]^{2},[u d][d s]_{+},[d s]^{2},[d s][s u]_{+}$, $[s u]^{2}$, and $[s u][u d]_{+}$combining with the remaining antiquark which give 18 pentaquark states in $\mathbf{8}_{F}$ plus $\overline{\mathbf{1 0}}_{F}$. All these states are degenerate in the $\mathrm{SU}(3)_{F}$ limit.

\section{The EH approach and the results}

The EH for the three constituents has the form

$$
H=\sum_{i=1}^{3}\left(\frac{m_{i}^{2}}{2 \mu_{i}}+\frac{\mu_{i}}{2}\right)+H_{0}+V
$$

where $H_{0}$ is the kinetic energy operator, $V$ is the sum of the perturbative one-gluon exchange potentials and the string potential $V_{\text {string. }}$.

The dynamical masses $\mu_{i}$ (analogues of the constituent ones) are expressed in terms of the current quark masses $m_{i}$ from the condition of the minimum of the hadron mass $M_{H}^{(0)}$ as function of $\mu_{i}{ }^{2)}$ :

$$
\frac{\partial M_{H}^{(0)}\left(m_{i}, \mu_{i}\right)}{\partial \mu_{i}}=0, \quad M_{H}^{(0)}=\sum_{i=1}^{3}\left(\frac{m_{i}^{2}}{2 \mu_{i}}+\frac{\mu_{i}}{2}\right)+E_{0}\left(\mu_{i}\right),
$$

$E_{0}\left(\mu_{i}\right)$ being eigenvalue of the operator $H_{0}+V$. Quarks acquire constituent masses $\mu_{i} \sim \sqrt{\sigma}$ due to the string interaction in (11). As of today the $\mathrm{EH}$ in the form of (1) does not include chiral symmetry breaking effects. A

2) Technically, this is done using the auxiliary field approach to get rid of the square root term in the Lagrangian [17, 18. Applied to the QCD Lagrangian, this technique yields the EH for hadrons (mesons, baryons, pentaquarks) depending on auxiliary fields $\mu_{i}$. In practice, these fields are finally treated as $c$-numbers determined from (2). 
possible interplay with these effects should be carefully clarified in the future.

The physical mass $M_{H}$ of a hadron is

$$
M_{H}=M_{H}^{(0)}+\sum_{i} C_{i}
$$

The (negative) constants $C_{i}$ have the meaning of the constituent self energies and are explicitly expressed in terms of string tension $\sigma$ [19]:

$$
C_{i}=-\frac{2 \sigma}{\pi \mu_{i}} \eta_{i}
$$

where

$$
\eta_{q}=1, \quad \eta_{s}=0.88, \quad \eta_{c}=0.234, \quad \eta_{b}=0.052
$$

In Eq. (5) $\eta_{s}, \eta_{c}$, and $\eta_{b}$ are the correction factors due to nonvanishing current masses of the strange, charm and bottom quarks, respectively. The self-energy corrections are due to constituent spin interaction with the vacuum background fields and equal zero for any scalar constituent.

Accuracy of the EH method for the three-quark systems is $\sim 100 \mathrm{MeV}$ or better [15, 16]. One can expect the same accuracy for the diquarkdiquark-(anti)quark system.

Consider a pentaquark consisting of two identical diquarks with current mass $m_{[u d]}$ and antiquark with current mass $m_{\bar{q}}(q=d, s, c)$. In the hyperspherical formalism the wave function $\psi(\boldsymbol{\rho}, \boldsymbol{\lambda})$ expressed in terms of the Jacobi coordinates $\boldsymbol{\rho}$ and $\boldsymbol{\lambda}$ and can be written in a symbolical shorthand as

$$
\psi(\boldsymbol{\rho}, \boldsymbol{\lambda})=\sum_{K} \psi_{K}(R) Y_{[K]}(\Omega),
$$

where $Y_{[K]}$ are eigen functions (the hyperspherical harmonics) of the angular momentum operator $\hat{K}(\Omega)$ on the 6-dimensional sphere: $\hat{K}^{2}(\Omega) Y_{[K]}=-K(K+4) Y_{[K]}$, with $K$ being the grand orbital momentum. For identical diquarks, like $[u d]^{2}$, the lightest state must have a wave 
function antisymmetric under diquark space exchange. There are two possible pentaquark wave functions antisymmetric under diquark exchange, the first one (with lower energy) corresponding to the total orbital momentum $L=1$, and the second one (with higher energy) corresponding to $L=0$. For a state with $L=1, l_{\rho}=1, l_{\lambda}=0$ the wave function in the lowest hyperspherical approximation $K=1$ reads:

$$
\psi=R^{-5 / 2} \chi_{1}(R) u_{1}(\Omega), \quad u_{1}(\Omega)=\sqrt{\frac{8}{\pi^{2}}} \sin \theta \cdot Y_{1 m}(\hat{\boldsymbol{\rho}})
$$

where $R^{2}=\rho^{2}+\lambda^{2}$. Here one unit of orbital momentum between the diquarks is with respect to the $\rho$ variable whereas the $\lambda$ variable is in an $S$-state. The Schrödinger equation for $\chi_{1}(R)$ written in terms of the variable $x=\sqrt{\mu} R$, where $\mu$ is an arbitrary scale of mass dimension which drops off in the final expressions, reads:

$$
\frac{d^{2} \chi_{1}(x)}{d x^{2}}+2\left[E_{0}+\frac{a_{1}}{x}-b_{1} x-\frac{35}{8 x^{2}}\right] \chi_{1}(x)=0,
$$

with the boundary condition $\chi_{K}(x) \sim \mathcal{O}\left(x^{7 / 2}\right)$ as $x \rightarrow 0$ and the asymptotic behavior $\chi_{1}(x) \sim \operatorname{Ai}\left(\left(2 b_{1}\right)^{1 / 3} x\right)$ as $x \rightarrow \infty$. In Eq. (8)

$$
\begin{aligned}
a_{1} & =R \sqrt{\mu} \cdot \int V_{\mathrm{C}}\left(\boldsymbol{r}_{1}, \boldsymbol{r}_{2}, \boldsymbol{r}_{3}\right) \cdot u_{1}^{2} \cdot d \Omega, \\
b_{1} & =\frac{1}{R \sqrt{\mu}} \cdot \int V_{\text {string }}\left(\boldsymbol{r}_{1}, \boldsymbol{r}_{2}, \boldsymbol{r}_{3}\right) \cdot u_{1}^{2} \cdot d \Omega,
\end{aligned}
$$

where

$$
V_{\mathrm{C}}\left(\boldsymbol{r}_{1}, \boldsymbol{r}_{2}, \boldsymbol{r}_{3}\right)=-\frac{2}{3} \alpha_{s} \cdot \sum_{i<j} \frac{1}{r_{i j}}
$$

and

$$
V_{\text {string }}\left(\boldsymbol{r}_{1}, \boldsymbol{r}_{2}, \boldsymbol{r}_{3}\right)=\sigma \cdot l_{\min }
$$

is proportional to the total length of the strings, i.e., to the sum of the distances of (anti)quark or diquarks from the string junction point. In the Y-shape, the strings meet at $120^{\circ}$ in order to insure the minimum energy. This shape moves continuously to a two-legs configuration where the legs 
meet at an angle larger than $120^{\circ}$. Explicit expression of $V_{\text {string }}\left(\boldsymbol{r}_{1}, \boldsymbol{r}_{2}, \boldsymbol{r}_{3}\right)$ in terms of Jacobi variables is given in [20].

The mass of the $\Theta^{+}$obviously depends on $m_{[u d]}$ and $m_{s}$. The current masses of the light quarks are relatively well-known: $m_{u, d} \approx 0, m_{s} \approx 170$ $\mathrm{MeV}$. The only other parameter of strong interactions is the effective mass of the diquark $m_{[u d]}$. In principle, this mass could be computed dynamically. Instead, one can tune $m_{[u d]}$ (as well as $m_{[u s]}$ and $m_{[d s]}$ ) to obtain the baryon masses in the quark-diquark approximation. We shall comment on this point later on.

In what follows, we use $\sigma=0.15 \mathrm{GeV}^{2}$, and explicitly include the Coulomb-like interaction between quark and diquarks with $\alpha_{s}=0.39$.

For the pedagogy, let us first assume $m_{[u d]}=0$. This assumption leads to the lowest $u u d d \bar{d}$ and $u u d d \bar{s}$ pentaquarks. If the current diquark masses vanish, then the $[u d]^{2} \bar{d}$ pentaquark is dynamically exactly analogous to the $J^{P}=\frac{1}{2}^{-}$nucleon resonance and $[u d]^{2} \bar{s}$ pentaquark is an analogue of the $J^{P}=\frac{1}{2}^{-} \Lambda$ hyperon, with one important exception. The masses of $P$-wave baryons calculated using the EH method acquire the (negative) contribution $3 C_{q}$ for $J^{P}=\frac{1}{2}^{-}$nucleons and $2 C_{q}+C_{s}$ for the $J^{P}=\frac{1}{2}^{-}$ hyperons. These contributions are due to the interaction of constituent spins with the vacuum chromomagnetic field. Using the results of Table 1 below we get the mass of the $P$-wave nucleon resonance with the orbital $\rho$ excitation $1600 \mathrm{MeV}$ and the mass of $\Lambda$ hyperon $1600 \mathrm{MeV}$ that within $100 \mathrm{MeV}$ agrees with the known $P$-wave $N$ and $\Lambda$ resonances.

However, the above discussion shows that the self-energies $C_{[u d]}$ equal zero for the scalar diquarks. This means that introducing any scalar constituent increases the pentaquark energy (relative to the $N$ and $\Lambda P$-wave resonances) by $2\left|C_{q}\right| \sim 200-300 \mathrm{MeV}$. Therefore prior any calculation we can put the lower bound for the pentaquark in the Jaffe-Wilczek approximation, $M(\Theta) \geq 2 \mathrm{GeV}$. 
The numerical calculation for $m_{[u d]}=0$ yields the mass of $[u d]^{2} \bar{s}$ pentaquark $\sim 2100 \mathrm{MeV}$ (see Table (1). The similar calculations yield the mass of $[u d]^{2} \bar{c}$ pentaquark $\sim 3250 \mathrm{MeV}$ (for $m_{c}=1.4 \mathrm{GeV}$ ) and $[u d]^{2} \bar{b}$ pentaquark $\sim 6509 \mathrm{MeV}$ (for $m_{b}=4.8 \mathrm{GeV}$ )[21]. For illustration of accuracy of the auxiliary field (AF) formalism in Table 1 are also shown the masses of $[u d]^{2} \bar{d}$ and $[u d]^{2} \bar{d}$ pentaquarks calculated using the spinless Salpeter equation (SSE):

$$
\begin{aligned}
H_{S} & =\sum_{i=1}^{3} \sqrt{\boldsymbol{p}_{i}^{2}+m_{i}^{2}}+V, \\
M & =M_{0}-\frac{2 \sigma}{\pi} \sum_{i=1}^{3} \frac{\eta_{i}}{\left\langle\sqrt{\boldsymbol{p}_{i}^{2}+m_{i}^{2}}\right\rangle},
\end{aligned}
$$

where $V$ is the same as in Eq. (11), $M_{0}$ is the eigenvalue of $H_{S}$, $\left\langle\sqrt{\boldsymbol{p}_{[u d]}^{2}+m_{[u d]}^{2}}\right\rangle,\left\langle\sqrt{\boldsymbol{p}_{\bar{q}}^{2}+m_{\bar{q}}^{2}}\right\rangle$ are the average kinetic energies of diquarks and an antiquark and $\eta_{i}$ are the correction factors given in (55). The numerical algorithm to solve the three-body SSE is based on an expansion of the wave function in terms of harmonic oscillator functions with different sizes [22]. In fact to apply this techniques to the three-body SSE we need to use an approximation of the three-body potential $V_{\text {string }}$ by a sum of the two- and one-body potentials, see [23]. This approximation, however, introduces the marginal correction to the energy eigenvalues. The quantities $\mu_{[u d]}$ and $\mu_{q}$ denote either the constituent masses calculated in the AF formalism using Eq. (44) or $\left\langle\sqrt{\boldsymbol{p}_{[u d]}^{2}+m_{[u d]}^{2}}\right\rangle,\left\langle\sqrt{\boldsymbol{p}_{\bar{q}}^{2}+m_{\bar{q}}^{2}}\right\rangle$ found from the solution of SSE. It is seen from Table 1 that these quantities agree with accuracy better than $5 \%$. The pentaquark masses calculated by the two methods differ by $100 \mathrm{MeV}$ for $\left([u d]^{2} \bar{s}\right)$ and $160 \mathrm{MeV}$ for $[u d]^{2} \bar{d}$. The approximation of $V_{\text {string }}$ mentioned above introduces the correction to the energy eigenvalues $\leq 30 \mathrm{MeV}$, so we conclude that the results obtained using the $\mathrm{AF}$ formalism and the SSE agree within $\sim 5 \%$, i.e., the accuracy of the AF results for pentaquarks is the same as for the $q \bar{q}$ system (see, e.g., [24]). 
If we withdraw an assumption $m_{[u d]}=0$, then a possible way to estimate the current diquark masses is to tune $m_{[u d]}, m_{[u s]}$ and $m_{[d s]}$ from the fit to the nucleon and hyperon masses (in the quark-diquark approximation). In this way one naturally obtains larger pentaquark masses. We have performed such the calculations using the SSE. We briefly investigated the sensitivity of the pentaquark mass predictions to the choice of $\sigma$, the strange quark mass $m_{s}$ and diquark masses $m_{[u d]}$ and found $M\left([u d]^{2} \bar{d}\right)$ in the range $2.2-2.4 \mathrm{GeV}, M\left([u d]^{2} \bar{s}\right) \sim 2.4 \mathrm{GeV}$ and $M\left([u s]^{2} \bar{d}\right) \sim 2.5 \mathrm{GeV}$.

Increasing $\alpha_{s}$ up to 0.6 (the value used in the Capstick-Isgur model [25]) decreases the $[u d]^{2} \bar{s}$ mass by $\sim 120 \mathrm{MeV}$ (see Table 21). We have briefly investigated the effect of the hyperfine interaction due to the $\sigma$ meson exchange between diquarks and strange antiquark and found that it lowers the $\Theta^{+}$energy by $\sim 180 \mathrm{MeV}$ for $g_{\sigma}^{2} / 4 \pi \sim 1$. As the result we obtain the lower bound of $[u d]^{2} \bar{s}$ pentaquark $M\left([u d]^{2} \bar{s}\right)=1740 \mathrm{MeV}$ (for $m_{[u d]}=0$ ) which is still $\sim 200 \mathrm{MeV}$ above the experimental value.

\section{Conclusions}

We therefore conclude that the string dynamics alone in its simplified form predicts too high masses of pentaquarks. This may indicate on a large role of the chiral symmetry breaking effects in light pentaquark systems. An "extremal" approach of chiral soliton model totally neglects the confinement effects and concentrates on the pure chiral properties of baryons. Therefore the existence of $\Theta$, if confirmed, provides an unique possibility to clarify the interplay between the quark and chiral degrees of freedom in light baryons.

This work was supported by RFBR grants No. 03-02-17345, 04-0217263, the grant for leading scientific schools No. 1774.2003.2. The NATO is also greatly acknowledged for the grant No. PST.CLG.978710. 


\section{References}

[1] T. Nakano et al. (LEPS Collab.), Phys. Rev. Lett. 91, 012002 (2003); hep-ex/0301020.

[2] V. V. Barmin et al. (DIANA Collab.), Yad. Fiz. 66, 1763 (2003) [Phys. At. Nucl. 66, 1715 (2003)], hep-ex/0304040.

[3] S. Stepanyan (CLAS Collab.), Phys. Rev. Lett. 91, 252001 (2003), hep-ex/0307018; V. Kubarovsky and S. Stepanyan (CLAS Collab.), hep-ex/0307088; J. Barth et al. (SAPHIR Collab.), Phys. Lett. B 572, 127 (2003); hep-ex/0307083; A. E. Asratyan, A. G. Dolgolenko, and M. A. Kubantsev, Phys. Atom. Nucl. 67, 682 (2004) [Yad. Fiz. 67, 704 (2004)], hep-ex/0309042; A. Airapetian et al. (HERMES Collab.), Phys. Lett. B 585, 213 (2004), hep-ex/0312044.

[4] D. Diakonov, V. Petrov, and M. V. Polyakov, Z. Phys. A 359, 305 (1997); hep-ph/9703373.

[5] C. Alt et al. (NA49 Collab.), Phys. Rev. Lett. 92, 042003 (2004), hep-ex/0310014.

[6] H1 Collab., Phys. Lett. B 588, 17 (2004), hep-ex/0403017.

[7] R. Matheus et al., Phys. Lett. B 578, 323 (2004), hep-ph/0309001; J. Sugiyama, T. Doi, and M. Oka, Phys. Lett. B 581, 167 (2004), hep-ph/0309271.

[8] F. Csikor et al., JHEP 0311, 070 (2003); S. Sasaki, hep-lat/0310014.

[9] Fl. Stancu and D. O. Riska, Phys. Lett. B 575, 242 (2003), hep-ph/0307010.

[10] M. Karliner and H. J. Lipkin, hep-ph/0307243.

[11] F. Huang et al., Phys. Lett. B 586, 69 (2004), hep-ph/0310040. 
[12] R. L. Jaffe and F. Wilczek, Phys. Rev. Lett. 91, 232003 (2003), hep-ph/0307341.

[13] For a review see Yu. A. Simonov, in Proceedings of the XVII Autumn School Lisboa, Portugal, 24 Sept. - 4 Oct. 1999, ed. by L. Ferreira, P. Nogueira, and J. I. Silva-Marco (World Sci., Singapore, 2000), p.60; hep-ph/9911237.

[14] I. M. Narodetskii, Yu. A. Simonov, M. A. Trusov, and A. I. Veselov, Phys. Lett. B 578, 318 (2004).

[15] Yu. A. Simonov, Phys. Atom. Nucl. 66, 338 (2003) [Yad. Fiz. 66, 363 (2003)]; hep-ph/0205334.

[16] I. M. Narodetskii and M. A. Trusov, Phys. Atom. Nucl. 67, 762 (2004) [Yad. Fiz. 67, 783 (2004)]; hep-ph/0307131.

[17] A. M. Polyakov, Gauge Fields and Strings (Harwood Academic Publishers, 1987).

[18] C. Semay, B. Silvestre-Brac, and I. M. Narodetskii, Phys. Rev. D 69, 014003 (2004).

[19] Yu. A. Simonov, Phys. Lett. B 515, 137 (2001).

[20] I. M. Narodetskii, A. N. Plekhanov, and A. I. Veselov, JETP Lett. 77, 58 (2003).

[21] A. I. Veselov (private communication).

[22] P. Nunberg, D. Prosperi, and E. Pace, Nucl. Phys. A 285, 58 (1977); B. Silvestre-Brac, R. Bonnaz, C. Semay, and F. Brau, Quantum threebody problems using harmonic oscillator bases with different sizes, internal report No. ISN-00-66, ISN, Grenoble, 2000 (unpublished).

[23] B. Silvestre-Brac, C. Semay, I. M. Narodetskii, and A. I. Veselov, Eur. Phys. J. C 32, 385 (2003). 
[24] V. L. Morgunov, A. V. Nefediev, and Yu. A. Simonov, Phys. Lett. B 459, 653 (1999).

[25] S. Capstick and N. Isgur, Phys. Rev. D 34, 2809 (1986). 


\section{Tables}

Table 1. The pentaquark masses in the quark-diquark-diquark approximations. The masses of $[u d]^{2} \bar{q}$ states $(q=d, s)$ for $J^{P}=\frac{1}{2}^{+}$pentaquarks are shown in units of $\mathrm{GeV}$.

\begin{tabular}{|c|c|c|c|c|}
\hline & & $\mu_{[u d]}$ & $\mu_{q}$ & $M$ \\
\hline$[u d]^{2} \bar{s} \frac{1^{+}}{2}$ & $\mathrm{AF}$ & 0.482 & 0.458 & 2.171 \\
& $\mathrm{SSE}$ & 0.463 & 0.468 & 2.070 \\
\hline$[u d]^{2} \bar{d} \frac{1^{+}}{2}$ & $\mathrm{AF}$ & 0.476 & 0.415 & 2.091 \\
& $\mathrm{SSE}$ & 0.469 & 0.379 & 1.934 \\
\hline
\end{tabular}

Table 2. The $[u d]^{2} \bar{s}$ mass calculated using the SSE as a function of $\alpha_{s}$ with and without the Goldstone boson exchange (GBE) in units of GeV.

\begin{tabular}{|c|c|c|c|}
\hline$\alpha_{s}$ & 0.39 & 0.50 & 0.60 \\
\hline$M$ (with GBE) & 1.893 & 1.814 & 1.737 \\
\hline$M$ (without GBE) & 2.069 & 2.007 & 1.949 \\
\hline
\end{tabular}

